\title{
Evaluación de la remoción de nutrientes y compuestos organoclorados y sus rutas de bioacumulación con la planta flotante Eichhornia crassipes expuesta a efluentes de pulpa de celulosa
}

\author{
Evaluation of nutrient and chlorinated organics removal \\ and their bioaccumulation pathways with the floating plant \\ Eichhornia crassipes exposed to pulp mill effluents
}

\begin{abstract}
Míguez, Diana (1), Martínez-Bengochea, Anabel (1), Carrara, María Victoria (1), Bombi, Katherine (2), Ferreira, Natalia (2), Cartmell, Elise ${ }^{(3)}$.

(1)Departamento Aguas y Productos Químicos, Laboratorio Tecnológico del Uruguay, LATU - (2) Departamento Aguas, Unidad Fray Bentos, Laboratorio Tecnológico del Uruguay, LATU. Parque Industrial, Barrio Anglo, Fray Bentos, Río Negro, Uruguay - (3) Cranfield Water Science Institute, Cranfield University, Bedfordshire, Reino Unido.
\end{abstract}

Contacto: dmiguez@latu.org.uy

RECIIDO: 30/5/2014 - APROBADO: 20/11/2014

\begin{abstract}
Resumen
El presente trabajo piloto tuvo como objetivo estudiar el proceso de bioacumulación de nutrientes y compuestos organoclorados en Eichhornia crassipes expuesta a efluentes de una fábrica de celulosa que vierte en aguas del Río Uruguay, cerca de la ciudad de Fray Bentos, Río Negro, Uruguay. Se caracterizaron los principales atributos del efluente, antes y después del cultivo con Eichhornia, así como también se estudiaron las concentraciones de los halógenos orgánicos en plantas control y en plantas sometidas al efluente. Se observaron cambios macroscópicos en la planta, los compuestos organoclorados se bioacumularon principalmente en las hojas, registrándose valores de $9,4 \mu \mathrm{g} / \mathrm{g}$ frente a $2,1 \mu \mathrm{g} / \mathrm{g}$ observados en la biomasa control. La remoción de nutrientes fue del orden del $90 \%$. Los resultados obtenidos sugieren que la fitorremediación con plantas flotables es un método potencialmente ventajoso para ser utilizado como tratamiento terciario en países tropicales y subtropicales para disminuir la concentración de compuestos orgánicos clorados recalcitrantes y como método de remoción de nitratos y fosfatos de efluentes de plantas de celulosa.

Palabras clave: Eichhornia crassipes, efluente, fitorremediación, halógenos orgánicos, bioacumulación.
\end{abstract}

\begin{abstract}
The aim of this pilot study was to evaluate the bioaccumulation processes of Eichhornia crassipes exposed to a secondary effluent obtained from a Kraft pulp mill that drains the effluents into the River Uruguay, near Fray Bentos city, Río Negro, Uruguay. The effluent was characterized before and after the Eichhornia culture, and organic halogens concentrations were determined in control and exposed specimens. Macroscopic changes were observed in the plant and the bioaccumulation routes were studied, the higher chlorinated organic compounds were found in the leaves (at a concentration of $9,4 \mu \mathrm{g} / \mathrm{g}$ by comparison to $2,1 \mu \mathrm{g} / \mathrm{g}$ of the control biomass). Nutrient removal was in the order of $90 \%$. The results suggest that phytoremediation with floating plants can be advantageously alternative as a tertiary treatment methodology in subtropical and tropical countries to diminish the concentration of recalcitrant chlorinated organics and also to remove nitrate and phosphate from pulp mill effluents.

Keywords: Eichhornia crassipes, effluent, phytoremediation, chlorinated organics, bioaccumulation.
\end{abstract}

\section{Introducción}

Las plantas flotantes representan importantes hábitats en los ríos y son fuente de alimento de macroinvertebrados, tales como larvas de insectos y crustáceos, peces, aves y mamíferos acuáticos. El género Eichhornia (o jacinto de agua) tiene una distribución muy extendida en cursos de agua del mundo, incluyendo el Río Uruguay. Se trata de una planta tropical 
flotante, considerada una especie invasora en diversas regiones del planeta (incluso en la región biogeográfica de origen). Sus tallos y raíces a menudo albergan bacterias fijadoras de nitrógeno, vertebrados e invertebrados, a veces en relaciones simbióticas o como fuente de alimento (Poi de Neiff, 2003; Ruiz Téllez, et al., 2008).

Se asume que las plantas acuáticas representan un componente clave de los humedales, ya que regulan los intercambios entre los sistemas terrestres y acuáticos, son capaces de asimilar directamente los compuestos potencialmente contaminantes, y promueven procesos de purificación aumentando la diversidad biológica y de reacciones químicas. Eichhornia crassipes es una especie muy utilizada en la construcción de humedales artificiales, dado que tiene un crecimiento rápido y la propiedad de almacenar nutrientes y contaminantes (Maine, et al., 2007). Por lo tanto, son plantas óptimas para tratamientos de fitorremediación, constituyendo una alternativa de bajo costo que puede ser utilizada para descontaminación de suelos y aguas (Pereira, et al., 2014).

El proceso de bioacumulación utilizando plantas es capaz de eliminar importantes porcentajes de metales pesados tóxicos de efluentes secundarios sin la producción de toxicidad, por lo cual esta técnica es muy recomendable para las aguas que se mezclan con efluentes industriales (Tripathi, et al., 2011), aunque se ha observado que ciertos metales tales como cobre, cobalto y cadmio pueden enlentecer su crecimiento (Buta, et al., 2011). Aprovechando su capacidad de asimilación de contaminantes, se han desarrollado distintos sistemas de tratamiento de efluentes industriales (como por ejemplo efluentes de papeleras) utilizando el jacinto de agua (E. crassipes) (Yedla, et al., 2002).

La aplicación de jacintos de agua para el uso del tratamiento terciario de efluentes de plantas de pulpa de celulosa con proceso Kraft es prometedora, puesto que, en condiciones experimentales, se han logrado eficiencias de remoción de materia orgánica de $46-75 \%$ y $11-17 \%$ de compuestos fenólicos totales (Lagos, et al., 2009). Además, por su capacidad de asimilación de nutrientes, se ha propuesto que E. crassipes se puede utilizar como una medida de remediación para los lagos eutróficos, controlando la producción de biomasa fitoplanctónica (Meerhoff, et al., 2002). Actualmente se cuenta con antecedentes científicos sobre el papel de E. crassipes en fitorremediación, constatándose la capacidad de estas especies de macrófitos acuáticos para disminuir metales pesados, como el cobre (Cu) y mercurio (Hg), de efluentes (Maine, et al., 2007; Mishra, et al., 2013). En este sentido, este último autor encontró una reducción de $\mathrm{Cu}$ del 63,6\% en el efluente de una papelera.

De acuerdo a estos antecedentes, se explora la utilización de Eichhornia crassipes como estrategia de fitorremediación y complementario al tratamiento químico convencional para eliminar contaminantes tóxicos de efluentes de la industria de la pulpa y el papel.

\section{Materiales y Métodos}

\section{Efluente}

Las muestras de efluente se obtuvieron de una fábrica ubicada en el bajo Río Uruguay cerca de la ciudad de Fray Bentos, Río Negro, Uruguay. Dicha planta industrial utiliza el proceso elemental Kraft libre de cloro para el blanqueo de celulosa.
El efluente se recogió a la salida del tratamiento secundario durante la producción de invierno del año 2009. Las principales características del efluente fueron: $\mathrm{DBO}_{5}$ de 29,0 mg/L (como $\mathrm{O}_{2}$ ), 0,563 mg/L de amoníaco (como N) y 1,84 mg/L de AOX (como Cl). La muestra del efluente también se caracterizó en términos de contenido de nutrientes (nitritos, nitratos, fósforo total y soluble), $\mathrm{pH}$, conductividad eléctrica y color (ver Tabla 1).

\section{Plantas}

Los especímenes de E. crassipes que se expusieron al efluente se recolectaron en un sitio aguas arriba de la planta de pulpa de celulosa (latitud: $33^{\circ} 5$ '35 .31" S; longitud: 58 $10^{\prime} 43.05^{\prime \prime} \mathrm{W}$ ) en octubre del año 2009. Como control negativo para el análisis de organoclorados acumulados en la planta se colectaron ejemplares de E. crassipes en el año 2006, antes de la puesta en marcha de la planta de celulosa. Se conservaron distintas partes de las plantas (tallo, hoja y raíces) congeladas a $-80{ }^{\circ} \mathrm{C}$ para analizarlas y comparar sus concentraciones con las muestras expuestas al efluente.

\section{Tratamientos}

En noviembre de 2009 los ejemplares de E. crassipes fueron expuestos al efluente por duplicado, utilizando régimen de lote, en tanques de polietileno de 20 litros conteniendo cinco litros de la muestra de efluente cada uno (Figura 1). La exposición fue de 31 días y se mantuvo en un lugar protegido dentro del laboratorio, con luz natural y a temperatura ambiente. Las pérdidas en volumen de cultivo debido a la evapotranspiración fueron contrarrestadas por adición de agua desionizada hasta el nivel original día por medio. Como control negativo se tomó una muestra de efluente (antes de comenzar con el ensayo) y se analizaron valores de conductividad, $\mathrm{pH}$, nitrito, nitrato, fósforo total y fósforo soluble, para poder ser comparados con los valores del efluente al terminar el ensayo.

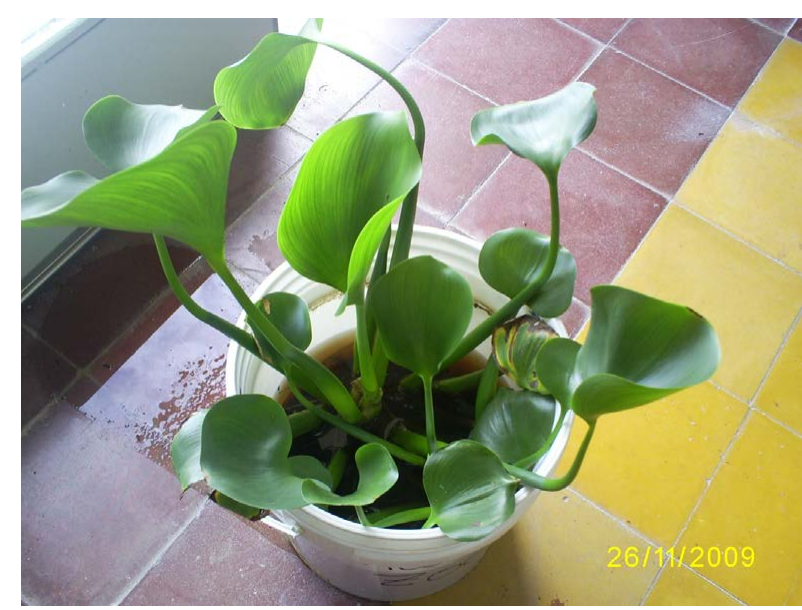

Figura 1. Eichhornia crassipes en presencia de efluente secundario al principio del ensayo.

\section{Reactivos}

Para los ensayos realizados por Soxhlet y EOX se utilizaron los siguientes reactivos: alcohol isopropílico: Mallinckrodt 
Químicos Lote H08B01 (ACS) 99,5\%, ciclohexano: Mallinckrodt Químicos Lote 4878KVVB 100\% puro y acetona: Merck KGaA 99,8\% puro.

Tras el período de exposición se cosecharon las plantas. Los tallos, hojas y raíces se separaron y trituraron utilizando un mortero y nitrógeno líquido. Su fracción lipófila se extrajo con $40 \mathrm{~mL}$ de una mezcla de ciclohexano:acetona (1:1) con un extractor líquido-líquido semi-automático (Soxtec System HT 1043, Tecator, Suecia).

Se pesaron las grasas obtenidas en cada porción de planta, se reconstituyeron con la mezcla de disolventes antes utilizada y se evaporaron bajo una corriente de nitrógeno. Los residuos evaporados se reconstituyeron en $0,5 \mathrm{~mL}$ de ciclohexano para finalmente medir EOX por el método de combustión/coulombimetría, utilizando un equipo Mitsubishi- COSA, modelo TOX-100.

También se determinaron los halógenos totales (TX). Las porciones trituradas de raíz, tallo y hoja se pesaron y quemaron directamente en el bote del equipo TOX-100 para la determinación de los halógenos totales (TX) por combustión/ coulombimetría.

El fósforo total y los fosfatos fueron analizados mediante el método de inyección en flujo con equipo Lachat 8500, de acuerdo a QuikChem Method 31-115-01-3-D (Lachat Instruments, 2009) y los nitritos y nitratos por el método QuikChem Method 10-107-04-1-A (Lachat Instruments, 2000). La conductividad se basó en la norma ISO 7888 (International Organization for Standarization, 1985). El pH se midió con electrodo, basándose en la norma APHA $4500 \mathrm{H}-\mathrm{B}$ (American Public Health Association, et al., 2001) y el $\mathrm{DBO}_{5}$ se determinó por el método respirométrico APHA $5210 \mathrm{D}$ (American Public Health Association, et al., 2001).

\section{Resultados}

Durante el desarrollo del experimento, el color de las hojas se tornó verde brillante, con un desarrollo importante de la planta. Después de una semana, los tallos expuestos mostraron signos de nodulación en los pecíolos, característica típica de la especie estudiada, mientras que en otras plantas no se observó ensanchamiento del pecíolo. El peso promedio inicial de la planta fue de $497 \pm 311 \mathrm{~g}$ y la masa vegetal final fue de $277 \pm 256 \mathrm{~g}$, peso húmedo.

Se observaron anfípodos, posiblemente del género Hyale$l l a$, viviendo en las raíces.

En este marco experimental, la absorción de nutrientes por parte de las plantas se evidencia por la disminución de fósforo y nitrato en los efluentes (Tabla 1).

Se utilizó la medida de color como una herramienta de monitoreo durante la prueba para analizar el efluente. El comportamiento de la variación del color fue similar en ambos duplicados (Gráfico 1).

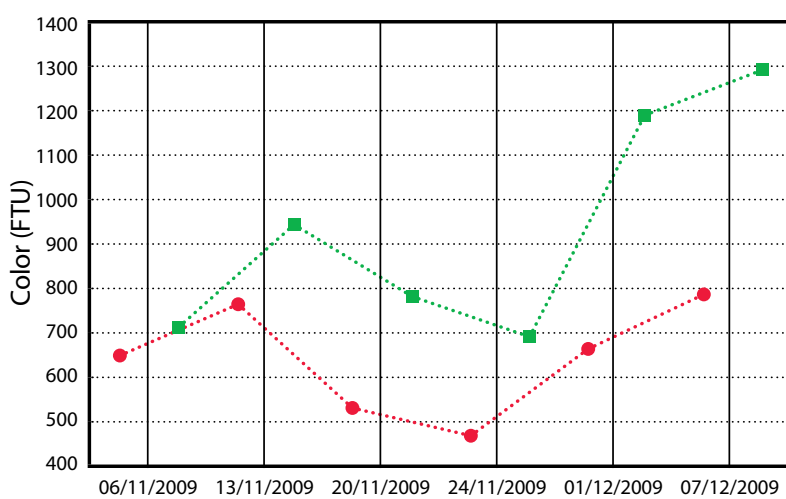

Fecha de exposición

- Planta 1

Gráfico 1. Análisis de color del efluente secundario en el transcurso de la exposición de E. crassipes.

\begin{tabular}{|l|c|c|c|}
\hline Parámetros & Valor previo a la exposición & Valor posterior a la exposición & Variación (\%) \\
\hline Conductividad $(\mu \mathrm{S} / \mathrm{cm})$ & 1326 & 5300 & -300 \\
$\mathrm{pH}$ & 7,18 & 7,80 & $-8,6$ \\
Nitrito (como N) (mg/L) & 0,31 & 0,072 & 76,8 \\
Nitrato (como N) (mg/L) & 1,34 & 0,016 & 98,8 \\
Fósforo total (como P) (mg/L) & 1,47 & 0,564 & 61,6 \\
Fósforo soluble (como P) (mg/L) & 1,47 & 0,095 & 93,5 \\
\hline
\end{tabular}

Tabla 1. Parámetros analizados en el efluente secundario, previo y luego de la exposición a la planta.

\begin{tabular}{|l|c|c|c|c|c|c|}
\hline \multirow{2}{*}{ E. crassipes } & \multicolumn{4}{|c|}{ Compuestos orgánicos halogenados extraíbles $(\mu \mathrm{g} / \mathrm{g})(\mathrm{como} \mathrm{Cl})$} & \multicolumn{4}{|c|}{ Compuestos halogenados totales $(\mu \mathrm{g} / \mathrm{g})(\mathrm{como} \mathrm{Cl})$} \\
\cline { 2 - 7 } & Raíces & Tallo & Hojas & Raíces & Tallo & Hojas \\
\hline No expuesto & 0,64 & 3,6 & 2,1 & 2,2 & 6,7 & 5,9 \\
Expuesto & 1,3 & 5,2 & 9,4 & 2,1 & 5,9 & 16,1 \\
\hline
\end{tabular}

Tabla 2. Análisis de halógenos orgánicos extraíbles y halógenos totales en plantas de E. crassipes que fueron expuestas y no expuestas al efluente secundario. 


\section{Eliminación de compuestos orgánicos clorados}

La ruta de distribución de compuestos orgánicos clorados existentes en el efluente de la fábrica de pulpa de celulosa se evidenció por un aumento en la concentración desde la raíz hacia las hojas (Tabla 2 y Gráfico 2).

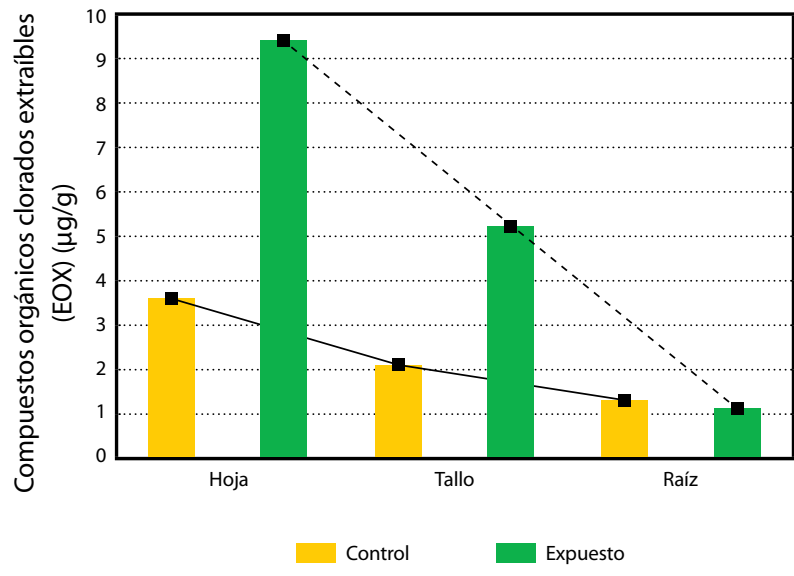

Gráfico 2. Distribución de halógenos orgánicos extraíbles (EOX) en diferentes tejidos (hojas, tallo y raíces) de plantas expuestas y no expuestas al efluente secundario.

\section{Discusión}

En este trabajo se demuestra que E. crassipes es capaz de absorber compuestos orgánicos halogenados y reducir la concentración de nutrientes de los efluentes analizados. La eficiencia de remoción de nutrientes en nuestra experiencia (65\% y $78,0 \%$, respectivamente), en especial para nitrito, fue similar a la reportada por Maine et al. (2007), quienes obtuvieron un porcentaje del $98,8 \%$ para el nitrato y un $76,8 \%$ para el nitrito. De acuerdo a Gao et al. (2014) la remoción de nitrógeno puede deberse no solamente a los procesos de asimilación por parte de la planta, sino a transformación a través de nitrificación y desnitrificación acopladas que producirían nitrógeno gas.

Algunos de los factores que podrían haber afectado las concentraciones acuosas de las sustancias orgánicas existentes en el efluente ensayado incluyen la hidrólisis, el factor de partición agua/carbono orgánico, el ingreso en la planta y su metabolismo (Chuluun, et al., 2009). Para compuestos clorados de muy baja biodegradabilidad, como los esperados en el efluente de pulpa de celulosa, estos autores encontraron que la acumulación en otra especie de planta flotante (Acorus gramineus) se produciría principalmente en la raíz, mientras que en el presente trabajo se encontró la mayor proporción de compuestos clorados en las hojas.

Una de las posibles razones que explican la inhibición del crecimiento después de 20 días de exposición es la alta salinidad, como sugieren Sooknah y Wilkie (2004). Además, según estos autores, la reducción de nutrientes sigue una cinética de primer orden en cultivos de 31 días, discontinuos o por lotes, afectando la producción de biomasa.

En posteriores experiencias, aunque se conoce la poca degradabilidad de los compuestos a remover, se debería incluir un control sin exponer las plantas para evaluar la degradación de estos compuestos, escalar este experimento a piloto, pero con ingreso de los efluentes en forma continua (Govindaswamy, et al., 2011), y, finalmente, utilizar esta tecnología en humedales construidos (Olukanni y Kokumo, 2013) como tratamiento terciario de los efluentes considerados. Luego de haber realizado ese tratamiento depurador, la planta puede usarse como sustrato para producir bioetanol o biogás para producción de energía a partir de biomasa o para generar biofertilizantes por compostaje (Patel, 2012), entre otras aplicaciones. Deberá estudiarse el ciclo de vida total, incluyendo la posibilidad de generación de dioxinas de la quema de las plantas que contienen estos contaminantes a la hora de decidir la disposición final.

\section{Conclusiones}

En base a los resultados de eliminación de compuestos organoclorados y la alta remoción de nutrientes (en el orden de $90 \%$ en esta experiencia), se sugiere que este método sería ventajoso como tratamiento terciario de efluentes de pulpa de celulosa. Las rutas de distribución de organoclorados dentro de la planta demostraron su acumulación principalmente en las hojas. El presente trabajo es el punto de partida para continuar con una línea de investigación de aplicaciones tecnológicas de bajo costo y ambientalmente sustentables para disminuir el aporte de nutrientes hacia cursos de agua desde fuentes puntuales industriales y como medida de mitigación de los riesgos de contaminación debidos a sustancias organocloradas persistentes.

\section{Reconocimientos}

Se agradece al LATU y a la Universidad de Cranfield, ya que este trabajo fue realizado en el marco de la tesis doctoral de Diana Míguez.

\section{Referencias}

American Public Health Association, American Water Works Association, and Water Pollution Control Federation, 2001. Standard methods for the examination of water and wastewater. 20a ed. Washington: APHA. Standard Method $5210 \mathrm{D}$.

American Public Health Association, American Water Works Association, and Water Pollution Control Federation, 2001. Standard methods for the examination of water and wastewater. 20a ed. Washington: APHA. Standard Method $4500 \mathrm{H}^{+}-\mathrm{B}$.

Buta, E., Paulette, L., Mihăiescu, T., Buta, M., Cantor, M., 2011 The influence of heavy metals on growth and development of Eichhornia crassipes species, cultivated in contaminated water. En: Notulae Botanicae Horti Agrobotanici ClujNapoca, 39(2), pp.135-141.

Chuluun, B., Iamchaturapatr, J. y Seong Rhee, J., 2009. Phytoremediation of organophosphorus and organochlorine pesticides by Acorus gramineus. En: Environmental Engineering Research, 14(4), pp.226-236.

Gao, Y., Yi, N., Wang, Y., Ma, T., Zhou, Q., Zhang, Z., Yan, S., 2014. Effect of Eichhornia crassipes on production of 
$\mathrm{N} 2$ by denitrification in eutrophic water. En: Ecological Engineering, 68, pp.14-24.

Govindaswamy, S., Schupp, D.A. y Rock, S.A., 2011. Batch and continuous removal of arsenic using hyacinth roots. En: International Journal of Phytoremediation, 13(6), pp.513-527.

International Organization for Standardization, 1985. ISO 7888. Water quality. Determination of electrical conductivity. Ginebra: ISO.

Lachat Instruments, 2000. Determination of nitrate/nitrite in surface and wastewaters by flow injection Analysis. Milwaukee: Lachat Instruments. Method 10-107-04-1-A.

Lachat Instruments, 2009. Determination of total phosphorus in brackish and fresh waters by flow Injection analysis. Milwaukee: Lachat Instruments. Method 31-115-01-3-D.

Lagos, C., Urrutia, R., Decap, J., Martínez, M. y Vidal, G., 2009. Eichhornia crassipes used as tertiary color removal treatment for Kraft Mill effluent. En: Desalination, 246, pp.45-54.

Maine, M.A., Suñe, N., Hadad, H., Sanchez, G. y Bonetto, C., 2007. Removal efficiency of a constructed wetland for wastewater treatment according to vegetation dominance. En: Chemosphere, 68, pp.1105-1113.

Meerhoff, M., Rodríguez-Gallego, L. y Mazzeo, N., 2002. Potencialidades y limitaciones del uso de Eichhornia crassipes (Mart.) Solms en la restauración de lagos hipereutróficos subtropicales. En: Fernández, A. y Chalar, G., eds. Agua en Iberoamérica: de la limnología a la gestión en Sudamérica. Buenos Aires: CYTED XVII. pp.61-74.

Mishra, S., Mohanty, M., Pradhan, C., Patra, H.K., Das, R. y Sahoo, S., 2013. Physico-chemical assessment of paper mill effluent and its heavy metal remediation using aquatic macrophytes: A case study at JK Paper Mill, Rayagada,
India. En: Environmental Monitoring and Assessment, 185, pp.4347-4359.

Olukanni, D.O. y Kokumo, K.O., 2013. Efficiency assessment of a constructed wetland using Eichhornia crassipes for wastewater treatment. En: American Journal of Engineering Research (AJER), 2(12), pp.450-454.

Patel, S., 2012. Threats, management and envisaged utilizations of aquatic weed Eichhornia crassipes: an overview. En: Reviews in Environmental Science and Bio/Technology, 11(3), pp.249-259.

Pereira, F., De Castro, E.M., De Olivera, C., Pires, M.F., Pereira, M.P., Ramos, S.J. y Faquin V., 2014. Lead tolerance of water hyacinth (Eichhornia crassipes Mart. - Pontederiaceae as defined by anatomical and physiological traits. En: Anais da Academia Brasilera de Ciencias, 86(3), pp.1423-1433.

Poi de Neiff, A., 2003. Macroinvertebrates living on Eichhornia azurea Kunth in the Paraguay River. En: Acta Limnológica Brasileira, 15(1), pp.55-63.

Ruiz Téllez, T., de Rodrigo López, E. M., Lorenzo Granado, G., Albano Pérez, E., Morán López, R. y Sánchez Guzmán, J.M., 2008. The water hyacinth, Eichhornia crassipes: an invasive plant in the Guadiana River Basin (Spain). En: Aquatic Invasions, 3(1), pp.42-53.

Sooknah, R.D. y Wilkie, A.C., 2004. Nutrient removal by floating aquatic macrophytes cultured in anaerobically digested flushed dairy manure wastewater. En: Ecological Engineering, 22, pp.27-42.

Tripathi, S. y Tripathi, B.D., 2011. Efficiency of combined process of ozone and bio-filtration in the treatment of secondary effluent. En: Bioresource Technology, 102(13), pp.6850-6.

Yedla, S., Mitra, A., Bandyopadhyay, M., 2002. Purification of pulp and paper mill effluent using Eichhornia crassipes. En: Environmental Technology, 23(4), pp.453-65. 